\title{
Physiological ecology of cerrado plants: new insights and new approaches
}

\author{
Augusto C. Franco ${ }^{1, *}$, Mundayatan Haridasan ${ }^{2}$ and Cristiane da Silva Ferreira ${ }^{1}$ \\ ${ }^{1}$ Universidade de Brasília, Instituto de Ciências Biológicas, Departamento de Botânica, CP 04457, Brasília DF 70904-970, \\ Brazil. ${ }^{2}$ Universidade de Brasília, Instituto de Ciências Biológicas, Departamento de Ecologia, CP 04457, Brasília DF 70904- \\ 970, Brazil. *Corresponding author: acfranco@unb.br
}

Received: 17 December 2008; Accepted: 17 December 2008

The cerrado vegetation of central Brazil is the second most extensive plant formation in South America. It is subject to regular and predictable annual drought, which is a major determinant of the ecosystem structure and function. High irradiance and air temperature, coupled with low relative humidity, impose a consistently high evaporative demand during the prolonged dry season. Nutrient-poor, acid soils with high levels of exchangeable aluminum cover most of the region and represent an additional limiting factor for plant growth. In spite of these strong constraints, the cerrado vegetation is remarkably speciesrich and has a complex community structure. The rich flora has a variety of adaptive strategies to stride over the long dry season, to grow with very little nutrients and to survive in a fire-prone environment. This special issue of the Brazilian Journal of Plant Physiology provides new insights into how cerrado plants cope with various demands imposed by the environment. They also explore the mechanisms of physiological adaptations at different scales and draw attention to the importance of phenotypic plasticity in plant adaptation to the cerrado environment. Most of them are not reviews but rather a series of case studies. Each presents new information or adds new approaches and analyses of already published data sets. Together they represent the opinions of scientists with diverse expertise working at different levels of organization (organism, population, community and ecosystem) and temporal and spatial scales on cerrado ecophysiology.

The cerrado vegetation exhibits substantial diversity not only in terms of the numbers of species, but also their growth forms, phenological behaviors, and basic physiology. Bucci and coauthors make use of this diversity to explore responses to water availability, comparing the water relations, leaf and stem hydraulic properties of a number of cerrado tree species ranging in nature from evergreen to deciduous. Scholz et al. propose a conceptual model of interactions of biophysical, morphological and physiological traits in an attempt to explain determinants of hydraulic architecture and variations in water economy of cerrado trees. Zaidan and Carreira provide a comprehensive synthesis of available data on seed germination of herb, shrub and tree species. Haridasan cautions that concepts of nutrient deficiencies and toxicities, well established for cultivated plants, should not be extended to native species in natural ecosystems indiscriminately. Several adaptive mechanisms such as root dimorphism, nocturnal transpiration, resorption of nutrients before senescence of leaves, recycling of nutrients from litter and mycorrhyzal associations could be involved in making cerrado plant communities more efficient than cultivated plants in poor soils. The results of Miranda and Cirne indicate that fruits of some cerrado species can be good insulators for seeds during fires. However, the damage of viable seeds in fruits that are already open before the fires and the large number of fruits that do not open after the fires represent a considerable loss of the year's seed production. The other two studies (Lemos et al. and Hoffmann and Franco) rely on comparisons of savanna and forest species of the Brazilian cerrado to gain insight into the selective pressures and constraints that explain the diversity in plant form and function in cerrado ecosystems. The studies of Lemos Filho et al. are focused on different populations of a single legume tree species, Plathymenia reticulata, which was used as a model on identifying specific morphological and physiological traits related to adaptation to the habitats of origin of each population. On the other hand, Hoffmann and Franco have relied on congeneric species pairs, each of which contains one savanna and one forest species. They highlight the importance of considering phylogenetic information for the design and analysis of comparative studies. 
High diversity in cerrado plants is reflected in an array of adaptations to the savanna environment and the small collection of papers presented in this issue is not sufficient to cover all aspects of physiological and environmental controls on cerrado plants. However the authors present enough evidence that selective pressures impose strong constraints on functional trait variability, despite the high plant diversity in cerrado ecosystems. Even though these studies have to be extended to a larger number of species and life forms and evaluated across a large geographical range, we believe that these case studies demonstrate well the dynamic nature of physiological mechanisms and its interactions with plant structure, phenology, and ecosystems processes. Though we are still far from being able to define the complex nature of regulation of resource use by plant species and their interactions with the environment, it is our hope that the articles in this special issue would stimulate collaborative studies that extend across organizational levels of plant forms and functions. 